\title{
Narrativa
}

narrativa

Nuova serie

39 | 2017

Nuove frontiere del Sud

\section{Il Matri-archivio del Mediterraneo: l'opera femminile incondizionata}

\section{Silvana Carotenuto}

\section{(2) OpenEdition}

1 Journals

Edizione digitale

URL: https://journals.openedition.org/narrativa/730

DOI: $10.4000 /$ narrativa. 730

ISSN: 2804-1224

Editore

Presses universitaires de Paris Nanterre

\section{Edizione cartacea}

Data di pubblicazione: 1 décembre 2017

Paginazione: 179-187

ISBN: 978-2-84016-289-6

ISSN: 1166-3243

Notizia bibliografica digitale

Silvana Carotenuto, «ll Matri-archivio del Mediterraneo: I'opera femminile incondizionata», Narrativa

[Online], 39 | 2017, online dal 01 décembre 2021, consultato il 14 janvier 2022. URL: http://

journals.openedition.org/narrativa/730 ; DOI: https://doi.org/10.4000/narrativa.730

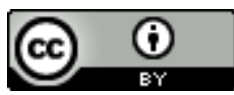

Narrativa est mise à disposition selon les termes de la Licence Creative Commons Attribution 4.0 International. 


\title{
Il Matri-archivio del Mediterraneo: l'opera femminile incondizionata ${ }^{1}$
}

\author{
L'idea poetica, per me, non si ferma alle frontiere. \\ Ogni poesia mediterranea è mia. \\ Ogni poesia che annuncia il viaggio, che dà ospitalità, che semina generosità. \\ In ogni angolo del mondo. È lì che comincia il Mediterraneo. \\ Non un luogo recintato da principi geografici o da un'idea
}

che rinnega l'Altro che viene dal sud, da Oriente o da Occidente; piuttosto un'idea aperta, dimora poetica...

Mohammed Bennis, Il Mediterraneo e la parola

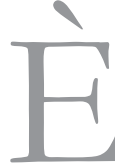

possibile un pensiero "femminile" sul Sud? È possibile che esso si dia in "prassi" di conservazione, performatività ed evento, in un Sud liquido, marino, mediterraneo? Il M.A.M, il Matri-archivio del Mediterraneo, vorrebbe testimoniare questa possibilità, ispirandosi a due testi di Jacques Derrida che si sono dimostrati centrali all'ideazione, prima, e alla realizzazione, dopo, del suo progetto: L'università senza condirzione, e Mal d'archivio ${ }^{2}$.

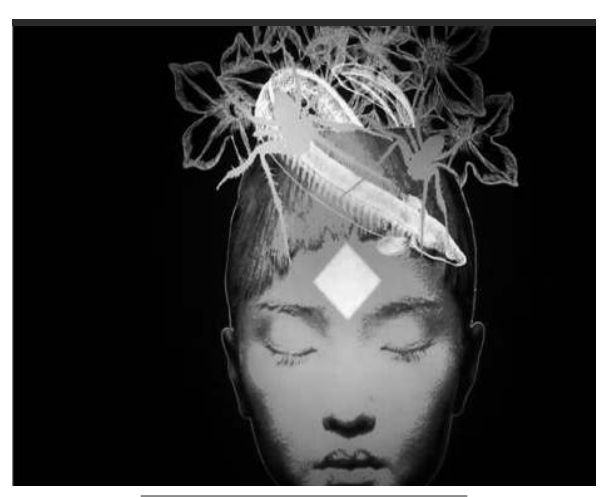

Antonia Bonetti, Sleeping Venus

( Courtesy dell'artista)

1. La piattaforma archivistica Matri-archivio del Mediterraneo. Grafie e Materie è il risultato della ricerca dal titolo originario L'archivio della performance femminile in area Mediterranea. Prove Digitali, condotta nell'a.a. 2013-2014 dalla Research Unit, costituita inizialmente da Beatrice Ferrara, Celeste Ianniciello e Annalisa Piccirillo, a cui si sono aggiunte poi Emanuela Esposito e Roberta Colavecchio, coordinata da Silvana Carotenuto (Dipartimento di Scienze Umane e Sociali, dell'Università degli Studi di Napoli "L'Orientale"); realizzazione grafica e sviluppo software: "Intuizioni Creative" (referente Alessandro Ventura). Il progetto ha beneficiato del finanziamento P.O.R. Campania FSE 2007-2013, Asse IV, Capitale Umano (www.matriarchiviomediterraneo.org).

2. Derrida, Jacques, Rovatti, Pier Aldo, L'università senza condizione, Milano, Cortina, 2002; Derrida, Jacques, Mal d'archivio. Un'impressione freudiana, Napoli, Filema, 1996. 
Nel testo sull'università, il filosofo franco-algerino afferma, a proposito della sua decostruzione degli studi umanistici, che la loro professione accademica deve essere arricchita con la produzione di "opere". In effetti, secondo Derrida, anche se l'università non è incondizionata, lo statuto della "Letteratura", il suo "come se" letterario, deve far sì che l'accademia insista tanto nella fede, o belief, quanto nell'assoluta incondizionatezza della sua istituzione, producendo, oltre al "pensiero", degli atti veri e propri di intervento nel reale, di creazione di visioni, o oeuvres" (il senso derridaiano qui è dichiaratamente ispirato dall'ambito delle arti), condivise e aperte al giudizio del nuovo spazio pubblico globale. Dal canto suo, Mal d'archivio traccia la priorità incondizionata sia per il pensiero che per l'opera: l'archivio. Il "mal d'archivio" è imprescindibile; si può dubitare che esso sia inteso a ricordare e/o a dimenticare, ma non si può essere in dubbio sul fatto che tutti amino "archiviare", che tutti soffrano del "mal" d'archivio. L'archivio è il luogo deputato alla memoria, affidato agli arkēe-onti, nella selezione dei testi che costituiscono il canone, la monumentalità della cultura, la sovranità della nazione. L'unico archivio (l'archivio dell'Unico/Uno) è maschile, patriarcale e patrilineare: il lascito, l'eredità e la consegna di ciò che ripete, salvaguarda e trasmette l'ordine, il potere, la tradizione. Che cosa succederebbe, si chiede Derrida, se fossero le donne, invece, a farsi "arconti"? Se fossero loro a intervenire nelle pratiche archiviali? Se il femminile si assumesse il compito di riflettere sull'archivio come strumento di una creatività che viene dal passato, che è praticata e aggiornata nel presente, e che è inviata, nella sopravvivenza ai dolori e ai traumi della contemporaneità, nel futuro à-venir?

Per rispondere a queste domande, il pensiero incondizionato del M.A.M. studia l'archivio, e lo traduce in opera: una piattaforma digitale che offre visibilità a giovani artiste mediterranee che, lavorando in vari media e con varie tecniche, sfruttando le potenzialità di diverse "grafie" e "materie", assumono la funzione di "matri-arke $\vec{e}$ " dell'arte che, dal mare fluido del Sud del mondo, emerge oggi, a ridosso del passato e rivolto all'avvenire. Perché l'arte? Perché l'incondizionatezza del pensiero e della prassi d'ispirazione decostruttiva giace nel "come se", as if / comme si, che si inscrive - in un gesto di scrittura che può "restare puramente orale, vocale, musicale: ritmico o prosodico", grafico e materico - nella letteratura, nell'immaginazione, nella finzione, nella fantasia:

3. Cfr. Derrida, Jacques, Bennington, Geoffrey, Derridabase. Circonfessione, Roma, Lithos, 2008. Non è casuale, per ciò che segue, che il testo che appare come "banda" annessa in basso alla scrittura dell'amico Bennington sia dedicato al rapporto di Derrida con la "madre".

4. Derrida, Jacques, Il Monoliguismo dell'altro o la protesi d'origine, Milano, Cortina, 2004. 
la libertà incondizionata di dire tutto, ed il contrario di tutto; la professione incondizionata di fede nell'impegno riflessivo, e, insieme, l'opera creativa che è pensata, conservata, praticata e trasmessa dalle e per le donne.

\title{
Il Matri-archivio del Mediterraneo. \\ Grafie e Materie
}

Matri-archivio/Mediterraneo: la "m" è suono, cellula minima o atomo della/e lingua/e: la madre, la mer, $\mathrm{m} /$ other, la matrice, mater, matter, la mater-ia, i materia/li. L'italiano, il francese, l'inglese: quante lingue potrebbero far eco, rifrangersi in onda, fiorire insieme nei legni dei "m"andorli mediterranei? $?^{5}$ Già da sole, in verità, la lingua madre, e l'estraneità intima delle lingue veicolari, rivelano le "risonanze" rinnovate di questo archivio femminile...

\section{LA MADRE}

\author{
Creare è una forma di maternità: \\ educa, rende felici e adulti in senso buono \\ Anna Maria Ortese, "Dove il tempo è un altro", in Corpo Celeste
}

Il 9 giugno 2017, Daniela Brogi ha pubblicato nel blog "Le parole e le cose. Letteratura e realtà" il bell'articolo "Nel nome della madre. Per un nuovo racconto di formazione", a stralcio dell'introduzione del libro collettaneo Nel nome della madre. Ripensare le figure della maternità. L'impegno della pubblicazione recita:

5. Sulla questione della "lingua madre", il M.A.M si ispira a Derrida, il quale afferma: "la madre, come lingua materna, l'esperienza stessa dell'unicità assoluta che può soltanto essere sostituita perché è insostituibile, traducibile perché intraducibile, proprio in quanto è intraducibile [...] sostituibile solo lì dove non c'è posto unico che per lei" (DERRIDA, Jacques, Il monolinguismo, cit, p. 75); si ispira anche alla trattazione derridaiana della poetica di Adorno: "Il suo discorso dovrebbe risultare esemplare, oggi, per tutti colori che cercano, nel mondo, ma in particolare nell'Europa in costruzione, di definire un'altra etica o un'altra politica, un'altra economia, o anche un'altra ecologia della lingua: come coltivare la poeticità dell'idioma in generale, il suo presso di sé, il suo oikos, come salvare la differenza linguistica, regionale o nazionale, come resistere allo stesso tempo all'egemonia internazionale di una lingua di comunicazione [...], come opporsi all'utilitarismo strumentale di una lingua puramente funzionale e comunicativa, sen 2 a tuttavia cedere al nazionalismo, allo statal-nazionalismo o al sovranitarismo statal-nazionalista, senza prestare queste vecchie armi arrugginite alla reattività identitaria e a tutta la vecchia ideologia sovranitarista, comunicarista e diffenzialista? [...]. Sogno, idioma poetico, malinconia, abisso dell'infanzia, Abgrund der Kindheit che non è altro [...] che la profondità di un fondo (Grund) musicale, la risonanza segreta della voce o dei vocaboli che attendono in noi, come al fondo del primo nome proprio di Adorno, ma senza potere [...], vulnerabili" (ID., Il sogno di Benjamin, Milano, Bompiani, 2003, pp. 21-26). 
[...] volevamo sperimentare uno sguardo che trasformasse il mondo della madre in una avventura [...] volevamo trattare la madre come un'identità culturale e relazionale, non solo emotiva, invece che come un monumento muto, pauroso e ingombrante ${ }^{6}$.

Per il M.A.M, il pensiero relazionale è evocato da Hélène Cixous quando si occupa della storia mitica-mitologica di Demetra e Persefone, riferendosi a "la complicità, la solidarietà, l'amore tra la madre e la figlia, la lega madre-figlia che cerca di resistere alla violenza dei sovrani, dei capi fallocratici"' " La madre e la figlia e, tra di loro, la "riparazione fantasmatica" del mito: quante figure mitiche ispirano le giovani artiste del M.A.M.? Demetra e Kore: nel bellissimo La ragazza indicibile di Giorgio Agamben, è Monica Ferrando, l'artista, a mettere in opera l'ispirazione, la tecnica, la valenza creativa e creatrice del mito ${ }^{8}$; Iside (Madre Natura), la dea abbandonata da Kant in una semplice nota, che pure incarna la capacità sublime dell'amore di contro alla perdita traumatica'; la ribelle Antigone che, con voce tragica, dice di un diritto d'asilo e di sepoltura che, come comprende Judith Butler, cambierebbe le sorti della società intera ${ }^{10}$; le Veneri dipinte dai/nei "Lavis" di Colette Deblè, impregnate dal desiderio di riscrivere l'intera storia dell'arte" ; la regina Cleopatra, che rivive in "La Leopardessa" di Emily Dickinson, e che riappare in Luna crescente di Diana Abu-Jaber come la cuoca irachena Sirine, innamorata del giovane esiliato Hanif, ad inventare una contemporanea storia d'amore multietnico" ${ }^{12}$; ancora ed ancora, la sfinge "Baby

6. Brogi, Daniela, "Per un nuovo racconto di formazione", in http:/ /www.leparoleelecose.it/?p=27899. L'articolo è pubblicato anche in Brogi, D., DE Rogatis, T., Franco, C., Spera, L. (a cura di), Nel nome della madre, Roma, Del Vecchio Editore, 2017, pp. 9-19. Per il tempestivo riferimento bibliografico, ringrazio l'amico Roberto M. DainotTo.

7. Cixous, Hélène, Peinetures. Ecrits sur l'art, Paris, Hermann, 2010, p. 28 (mia traduzione).

8. Agamben, Giorgio, Ferrando, Monica, La ragazza indicibile. Mito e mistero di Kore, Milano, Electa, 2010. In specifico per l'arte di Ferrando, si rinvia a Carotenuto, Silvana, "Un evento di indicibilità: Quando il libro si fa... ragazza", in Leggendaria, vol. 15, 2011, pp. 52-54.

9. Cfr. Freeman, Barbara Claire, The Feminine Sublime. Gender and Excess in Women's Fiction, Berkeley, University of California Press, 1995, pp. 105-148.

10. Cfr. ButLer, Judith, Antigone's Claim. Kinship between Life and Death, New York, Columbia U. P., 2002.

11. Cfr. Derrida, Jacques, Prégrances. Lavis de Colette Deblé. Peintures, l'Atelier des Brisants, 2004. Interessante il film Lavis de Colette Deblé, realizzato dall'Université Lille 3 (https://youtu.be/JRYJ2tmFJ7w).

12. Cfr. Carotenuto, Silvana, La lingua di Cleopatra. Traduzioni e sopravvivenze decostruttive, Milano, Marietti, 2009 (il romanzo di Diana Abu-Jaber è pubblicato da 
Sugar" con cui l'artista Kara Walker incarna l'immensa memoria della schiavitù, rendendo responsabili noi tutti delle sue nefaste conseguenze ${ }^{13}$.

Il M.A.M. evoca la puissance di queste "matriarche" d'oggi e di molte altre ancora:

Alterità sublimi, sempre in bilico tra più mondi, universi e pianeti, dolci, sinuose, minacciose, gioiose, gloriose, splendidamente malinconiche come soli neri, le 'matriarche' incarnano una genealogia che nasce da acque tormentate da ostilità, guerre, morti, disfatte, per lasciare liberare il loro canto-inno-urlo di vita. È il perimento più ampio del Matriarchivio del Mediterraneo, la cornice di affetti che trasforma l'archiviazione classica perché la forza evocatrice, antica, sperimentale, materica e misteriosa delle matriarche spezza, frantuma, decostruisce l'Arké patriarcale, rilanciandolo, con la creazione d'una collettività impossibile, verso un futuro tutto da inventare, tutto $\grave{a}-v e n i r . .{ }^{14}$.

LA MER

\author{
Quel mare, il Mediterraneo, \\ è anche il grembo della nostra storia, \\ della nostra civiltà \\ Claudio Magris, "Per una filologia del mare"
}

In assonanza di lingua, la madre e la mer, il Mar Mediterraneo, il bacino di pace, di scambi culturali, di lingue e di poesia: il poeta Mohammed Bennis afferma che "Lo scambio creativo è la nostra salvezza"15. È il mare dei passaggi, dei transiti e delle migrazioni che provoca l'impegno poetico: Mona Hatoum, Zineb Sedira, Lara Baladi, Emily Jacir, Ursula Biemann hanno consegnato alla storia le loro visioni poetiche di questo mare fatto di desiderio, di fuga e di esilio, ponendo argine alla violenza che tinge di sangue le sue acque, con la ricerca indomita di pratiche artistiche che rielaborino gli esili, i viaggi, le diaspore passate e presenti ${ }^{16}$. Nella legacy di questi e di tanti altri progetti femminili, le giovani

Mondadori, 2001). Aggiungerei, qui, alla vastità e varietà di riscritture - shakespeariane e altre - il monologo Cleopatras di Giovanni Testori, nella straordinaria interpretazione dei Magazzini Criminali del 1996.

13. Il progetto è presentato on line (http://creativetime.org/projects/karawalker/).

14. In www.matriarchiviodelmediterraneo.org, sezione "Matriarca".

15. Bennis, Mohammed, Il Mediterraneo e la parola. Viaggio, poesia, ospitalità, Roma, Donzelli, 2009, p. 24.

16. Per la retrospettiva dell'opera di Mona Hatoum organizzata alla Tate Modern, Londra, 2016, vedi: http://www.artribune.com/report/2016/06/mostra-mona- 
artiste del M.A.M. aprono le loro preziose scatole di attrezzi artistici per esporre le estetiche della migrazione al focus di una creazione politico-poetica che, sfidando l'archiviazione neutra, di dati, nomi, corpi e bare, rivendica la trasformazione del trauma, del dolore e della perdita nell'appello ad una collettività di cura e d'attenzione, di testimonianza e di comunicazione, dell'esperienza viva e vitale delle donne, dei loro figli e dei loro amati, negli attraversamenti di un mare che sempre più si fa abisso di morte e, insieme, matrice di vita, di altra esistenza e resistenza, di nuove direzioni di senso ${ }^{17}$.

\section{LA MATRICE}

...un esperimento di scrittura per me è totale, è quello della vita Jacques Derrida, Sulla parola

Madre, utero, origine, e, allo stesso tempo, incavo e impronta; la matrice è ciò che è unico e singolare, e, allo stesso modo, riprodotto o riproducibile. La matrice tecnica è la vocazione del M.A.M. che avanza secondo una discendenza "matri-lineare" intesa quale creazione e passaggio della "linea", del segno, di quella organicità che, per l'artista Lygia Clark (che pensava e agiva da un altro Sud del mondo, il Brasile), è l'“incisione", il taglio sulla superfice piana della tela, la piega sui corpi materici delle opere ${ }^{18}$. La linea corre come una frontiera, disfacendo la piattezza del supporto materiale del dipinto o della scultura, e emergendo come spazio di ibridazione tanto concettuale quanto materiale:

La linea organica è una linea che non è stata abbozzata o incisa da nessuno, ma che risulta dal contatto di due superfici differenti (piani, cose, oggetti, corpi,

hatoum-tate-modern-londra/; per l'opera di Zineb Sedira, vedi: http://www.zinebsedira.com; per il lavoro transmediale Vox Populi, Archiving a Revolution in the Digital Age di Lada Baladi al MIT- Center for Art, Science \& Technology (CAST), vedi: https:// arts.mit.edu/artists/lara-baladi/\#about-the-residency; per il crowdfunding lanciato da Emily Jacir per trasformare la sua casa a Betlemme in centro d'arte, vedi: http:/ /www. artribune.com/arti-visive/street-urban-art/2017/05/emily-jacir-lancia-un-crowdfundingper-trasformare-la-sua-casa-a-betlemme-in-centro-darte/; per il progetto di Ursula Bieman, intitolato "Egyptian Chemistry" (2012), vedi: https://www.geobodies.org/ art-and-videos/egyptian-chemistry.

17. Cfr. Balzano, Wanda, Carotenuto, Silvana (a cura di), Anglistica-Aion, vol. 17.1, 2013. Questo numero è dedicato a "Writing Exile. Arts and Technologies of Women" (consultabile in http://sebinaol.unior.it/sebina/repository/catalogazione/ documenti/2013\%2017.1\%20ANGLISTICA\%20BALZANO\%20CAROTENUTO.pdf).

18. Cfr. Clark, Lydia, "The Abandonment of Art - 1948-1988”, la retrospettiva affidata al Moma di New York nel 2014 (consultabile all'indirizzo https://www.moma. org/calendar/exhibitions/1422). 
o anche concetti), essa annuncia un modo di pensare oltre la logica del vero o falso, $[. .$.$] punta ad un modo di pensare senza contraddizioni, senza dialettica [. .$. sebbene accetti la divergenza; pensiero affermativo [...]; pensiero del molteplice ${ }^{19}$.

La linea è la possibilità di superare ogni dialettica tra composizione e creazione, vero e falso, pubblico e partecipant, facendola esistere nell'incontro di due superfici; la linea esiste, è uno spazio in sé, costituendo la trasformazione del problema formale e astratto in materia, nella produzione di corpi, in forme di vita. È da essa che nascono ricerche innovative sul vuoto, sul piano e sulla superfice, sulla biodimensionalità, i silenzi, le membrane, le zone di contatto o interfacce, l'in-betweeness o border-line quali luoghi dell'evento e dell'invenzione, dove i processi creativi sono pensati e prodotti. Lo strumento di immaginifica rappresentazione della linea trova accoglienza di sperimentazione e di differance nel M.A.M., r-accogliendo la creatività femminile nel limite aperto tra discorso e visibilità, producendo la propria cartografia di forme, comunicando la politicità potenziale, concreta e dinamica, di corpi estetici le cui vibrazioni si mostrano permeabili e di membrana, capaci di assorbire forze ed effetti/affetti come elementi del tessuto partecipativo, marchi delle sensazioni che ne compongono la memoria.

$$
\begin{gathered}
\text { I MATER-IA/LI } \\
\text {... esplorare le possibilità che un riciclo creativo di approcci, metodologie, } \\
\text { idee, materie, corpi e pratiche di conoscenza, aperte all'aspirazione } \\
\text { e alla sperimentazione di nuovi modi di abitare gli spazi e i tempi contemporanei e à-venir. }
\end{gathered}
$$

Il gioco del M.A.M. è l'enfasi sulla materia - umana, animale, vegetale, tecnologica - in un gioco di frammentazione, aggiunta ed estensione: mater-ia/matters/materia/li. La materia porta dentro di sé il materno; aggiungendo la " $\mathrm{t}$ " a mater, la parola matter indica le "questioni", ciò che importa: "Bodies that matter/Postcolonial Matters" "20. Nella sezione dedicata a Matters, il M.A.M. crea una piattaforma, aperta e in infinito processo, dove è possibile condividere le priorità, i pensieri, le indicazioni teoriche, i motivi di riflessioni urgenti alla

19. Bausbam, Ricardo, "Within the Organic Line and After", in Alberro, Alexander, Buchmann, Sabeth (a cura di), Art after conceptual art, Cambridge, MA/London, MIT Press Vienna, Generali Foundation, 2006, pp. 87-99.

20. Butler, Judith, Bodies that Matter, New York and London, Routledge, 1993; Cianeldi, Alessandra, Ferrara, Beatrice (a cura di), Postcolonial Matters. Tra gesti politici e scritture poetiche, Napoli, Photocity, 2015. 
comunità che frequenta il suo archivio. Ciò che "riguarda" è, allo stesso tempo, e precisamente, il dibattito teorico e le sue concretizzazioni artistiche sull'uso, sul riuso e sul riciclaggio dei materiali di scarto, le sperimentazioni e gli sconfinamenti (di discipline, materie, materiali, corpi) che i linguaggi artistici propongono, ponendo in primo piano la questione del molteplice, di una complessità che non può che articolarsi nel superamento della dicotomia natura/cultura. La bio art contemporanea di firma femminile mette in campo questioni etiche, politiche e sociali, traducendo in arte responsabile il sentire, le problematiche e le contraddizioni del momento storico vissuto dalle donne.

Lo scenario sociale e culturale contemporaneo si fa espressione di un'attenzione ai temi dell'ecologia, al rapporto con la natura e con la materia. All'interno di un contesto globalizzato - in cui la circolazione di persone, beni e prodotti, replica i tempi accelerati della digitalizzazione di dati, corpi e informazioni - le artiste del M.A.M. s'interrogano sulle modalità etiche ed estetiche della relazione che intercorre tra l'umano, il naturale, l'animale, la tecnologia. Rintracciabile nelle pratiche e negli oggetti del vissuto quotidiano (i dispositivi tecnologici che mediano le relazioni sociali, i cibi geneticamente modificati e quelli di coltivazione biologica, le banche di semi, le droghe, i farmaci, gli esperimenti sugli animali...), la relazione tra umano, naturale, animale e tecnologia si disvela e si dispiega nei termini di una contaminazione molecolare, cellulare, atomica, quantistica. Ciò pone al centro della scena d'archiviazione l'interrogazione delle tradizionali concettualizzazioni essenzialistiche di una Natura ingabbiata nel gioco dicotomico con/tro la cultura, riconoscendo invece la queerness, la differenza, l'Alterità, l'eterogeneo che risiedono nel cuore della stessa: $M$-Other Nature.

\section{OSPITALITÀ $^{21}$}

Scrivere è chiedere ospitalità alla parola senza imporle di raccontarne noi stessi, ma accettando che essa racconti l'altro in noi Donatella Puliga, L'ospitalità è un mito?

Il M.A.M. "pensa" e "fa la verità" dei sensi dell'archiviazione decostruttiva: il "constativo", il "performativo" e la produzione dell'“evento". La piattaforma non vuole venir meno al "mal d'Africa" dell'archivio, identificato con la funzione

21. I testi che, in particolare, hanno ispirato il pensiero-opera d'ospitalità del M.A.M. sono: Derrida, Jacques, Dufourmantelle, Anne, Sull'ospitalità, Baldini\&Castoldi, 2000; Puliga, Donatella, L'ospitalità è un mito? Un cammino tra $i$ racconti del Mediterraneo e oltre, Genova, il Melangolo, 2010; Irigaray, Luce, L'ospitalità del femminile, Il nuovo Melangolo, Genova, 2014. 
constativa della conservazione - la registrazione di ciò che esiste, e che va protetto e conservato. Essa vuole, però, affiancarvi la vocazione performativa, con il sapere delle donne che non è mai statico: intervenendo nella consegna, il M.A.M. si apre alla performatività delle produzioni artistiche femminili che esistono sempre in sviluppo, in farsi, in progress e process, in divenire. Ancora, e forse più centralmente alla sua passione, oltre alla funzione constativa e all'interno del performativo, l'appello è rivolto all'inaspettato, all'imprevisto, all'inaudito, a cui ogni archivio del futuro dovrebbe esporre: l'infinita apertura, la metamorfosi incessante, la partecipazione - individuale e collettiva, singolare e metonimica - che l'archiviazione offre alle artiste, accoglie, conserva, e rilancia le condizioni dell'à-venir, di ciò che accade senza orizzonte di aspettativa, d'anticipazione e/o di previsione, che solo esiste nella chance della resistenza a ogni archiviazione: l'evento.

È all'insegna dell'evento che il M.A.M. interpella il materno nella fluidità del ventre marino; tra acque agitate, sullo sfondo di scenari naturali, il suo arkēè la vastità delle cave, dei cieli e delle terre che ne popolano l'universo acquatico; sospinto dai palpiti e dai respiri dei venti che spaziano nei suoi bacini, esso ibrida e confonde le lingue; la fede incondizionata resta nella creazione, nell'invenzione, nell'immaginazione che celebra l'amore delle donne, il desiderio di ospitarne l'arte, per dire e tramandare il "vieni" all'evento...

Viens, viens, ospite, guest/ host, perché il Matri-archivio del Mediterraneo vive e vivrà con il dono delle tue "opere"; artiste mediterranee, unitevi al M.A.M, affidando contributi, e contatti, alla sezione "Call for Proposal".

Per tutto, e oltre, il ringraziamento va alla "danzatrice" Annalisa Piccirillo e alla "curatrice" Celeste Ianniciello, che, con Beatrice Ferrara, Manuela Esposito e Roberta Colavecchio, hanno pensato e fatto il M.A.M; per tutte loro è la gratitudine, l'affetto, e la stima.

Silvana CAROTENUTO Università Napoli l'Orientale 
